\title{
Supportive care of cancer patients with a self-applied photobiomodulation device: a case series
}

\author{
Lilach Gavish $^{1,2}$ (D) Yehuda Zadik ${ }^{1,3} \cdot$ Rose Raizman ${ }^{4,5}$ \\ Received: 30 October 2020 / Accepted: 21 January 2021 / Published online: 30 January 2021 \\ (C) Springer-Verlag GmbH Germany, part of Springer Nature 2021
}

\begin{abstract}
Purpose Oral mucositis (OM) and prolonged wound healing are common side-effects of cancer treatments. Photobiomodulation (PBM), previously called low-level laser, is currently part of the official guideline for OM prevention. However, all the PBM protocols relate to office-based devices, operated by professional health caregivers, requiring frequent applications. In the following case series, we present our experience with a self-applied consumer home-use PBM device for supportive care.

Methods Five patients receiving cancer treatment presented at the clinic (female:male 3:2, 55-76 years old) with OM grade 3/4 $(n=2)$, post-surgical non-healing wounds $(n=3)$, and dermatitis $(n=1)$. The PBM treatment $(808 \mathrm{~nm}, 250 \mathrm{~mW}$ peak power, $15 \mathrm{KHz}, 5 \mathrm{~J} / \mathrm{min}$, ray size $4.5 \times 1.0 \mathrm{~cm}^{2}$ ) was self-applied by the patients. The protocol included extra/intra-oral applications, over the wound $\mathrm{bed} / \mathrm{margins}$ and adjacent lymph nodes.

Results The treatment was found effective for resolving OM with rapid pain relief and accelerated healing in post-operative wounds and dermatitis, without reported adverse events. Patients found routine easy to follow and painless, and the protocol was easily integrated as an adjuvant treatment to standard care at the clinic or home while not requiring additional time from the staff. Conclusions Side-effects induced by cancer therapy have a detrimental effect on the patient's well-being and may delay or even prevent the patients from completing treatment regimens. PBM is already an established tool for supportive treatment in cancer patients. The advent of a self-applied personal PBM treatment with easy-to-apply protocols for a variety of side-effects makes this technology an important accessible and safe supportive care option.
\end{abstract}

Keywords Dehiscence, Oral mucositis · Photobiomodulation $\cdot$ Low-level laser $\cdot$ Home-use $\cdot$ Supportive care $\cdot$ Case report

These case reports were presented as part of an oral presentation in March 2019 at the $39^{\text {th }}$ annual conference of the American Society for Lasers in Medicine and Surgery (ASLMS), Denver, Colorado, USA, in April 2019 at Wounds Canada spring conference, Halifax, Canada, and as an ePoster in June 2019 at the $29^{\text {th }}$ annual conference of the European Wound Management Association (EWMA), Gothenburg, Sweden.

Lilach Gavish

lilachg@ekmd.huji.ac.il

1 Institute for Research in Military Medicine (IRMM), Faculty of Medicine, The Hebrew University of Jerusalem, POB 12272, 9112001 Jerusalem, Israel

2 The Saul and Joyce Brandman Cardiovascular Research Hub, Institute for Medical Research (IMRIC), Faculty of Medicine, The Hebrew University of Jerusalem, POB 12272,

9112001 Jerusalem, Israel

3 Department of Oral Medicine, Sedation and Imaging, Hebrew University-Hadassah School of Dental Medicine, Jerusalem, Israel

4 Department of Professional Practice, Scarborough Health Network, Centenary Hospital, Toronto, Canada

5 Lawrence S. Bloomberg Faculty of Nursing, University of Toronto, Toronto, Canada

\section{Introduction}

In cancer therapy, chemotherapy and radiotherapy may damage healthy tissues leading to side-effects, among which oral mucositis (OM) [1] and prolonged wound healing are prominent [2]. OM is characterized by oral mucosal erythema, ulceration, and pain, thus frequently impairing the ability for food intake, and potentially preventing the patient from receiving the full cancer treatment regimen [1]. Prolonged wound healing may lead to postoperative complications in the form of dehiscence and increased incidence of infection, as well as the formation of non-healing chronic wounds and impaired quality of life [2].

Photobiomodulation (PBM), previously termed lowlevel laser therapy, is the application of non-ionizing visible or near-infrared optical radiation to tissue. The photons are absorbed by endogenous chromophores that elicit photochemical events without creating thermal damage [3, 4]. PBM is used clinically to accelerate 
wound healing and reduce inflammation, edema, and pain $[3,4]$ and was shown to affect multiple molecular pathways related to wound healing and to be particularly effective in the acceleration of healing of chronic wounds [5]. In the past 2 decades, PBM has been increasingly used as a new treatment modality in cancer patient supportive care and was suggested for prevention and management of cancer-induced toxicities [6-8] without association to long-term risk of malignant transformation, progression, or recurrence [9, 10]. International guidelines for the prevention of $\mathrm{OM}$ using PBM were published by the UK National Institute for Health and Care Excellence (NICE) interventional procedure guidance (IPG615)[11] and by the Multinational Association of Supportive Care in Cancer, and the International Society of Oral Oncology (MASCC/ ISOO)[7, 12].

However, the existing data originated from studies using office-based PBM devices at the clinic were operated by professional health caregivers. The requirement for multiple frequent treatment sessions (usually 2 to 5 sessions per week) at the clinic is in many cases not feasible nor convenient for the patients [13] and may also impose a serious health concern for these immunocompromised patients.

In the following case series, we present our experience with an approved (in Israel, Canada, Europe, and Brazil) consumer home-use near-infrared PBM device (B-Cure Laser Pro, Good Energies Ltd., Haifa, Israel). This lightweight, hand-held device was previously shown to be effective for the treatment of peri-implant gingival mucositis [14] and diabetic foot ulcers $[15,16]$. The energy parameters of the device are $808 \mathrm{~nm}$ wavelength, $250 \mathrm{~mW}$ peak power $\left(55 \mathrm{~mW} / \mathrm{cm}^{2}\right), 15 \mathrm{KHz}, 1.1$ $\mathrm{J} / \mathrm{cm}^{2} / \mathrm{min}$, while the ray size is $4.5 \times 1.0 \mathrm{~cm}^{2}$ enabling the simultaneous irradiation of a relatively large area in a short time compared to laser pointers.

We have developed a protocol based on our interpretation of the PBM literature related to the treatment of $\mathrm{OM}$ and other cancer-related side-effects [8] combined with our experience in the treatment of non-healing wounds [16], while taking into consideration the technical specifications of the device.

For OM, the protocol includes PBM irradiation intraorally over the tongue and inner epithelial surface of the lips, and extra-orally on the cutaneous surface corresponding to the buccal mucosa, and cervical lymph nodes. For non-healing post-operative wounds and dermatitis, the protocol includes irradiation over the wound bed, wound margins, tunneling wound (an internal channel that extends from the post-operative wound into the subcutaneous tissue), and adjacent lymph nodes.
Aim

Our purpose was to report cases demonstrating a significant improvement following self-applied PBM prophylaxis/ treatment for patients with complications of cancer therapy and to detail the protocol used.

\section{Cases}

Here we report 5 patients (3:2 female: male, with an age range of 55-76 years), of which, 2 had OM (grade 3 and 4, according to the World Health Organization [WHO] scale, i.e., oral ulcers were present and only liquid diet is possible, or oral alimentation impossible, respectively (Fig. 1)), 3 had post-operative wounds (post-Hartmann reconstruction and small bowel resection dehiscence (Fig. 2), non-epithelialized posthemicolectomy abdominal wound, post-total mastectomy seroma wound with tunnel), and one had chemotherapyinduced dermatitis in the groin area (and concomitant OM; (Fig. 3)). The treatments took place during MayAugust 2018 in 5 consecutive patients that were referred to the clinic and agreed to therapy (additional 2 patients who were presented to the clinic during this period with cancer treatment-related side-effects and were offered to be treated with PBM refused to be treated with this method). The patients' relevant medical background, specific PBM treatment protocols, and responses are summarized in Table 1. The detailed timeline of case $\# 1$ is presented in Table 2. This case series was written according to the CARE reporting guidelines [17].

\section{Discussion}

In the cases presented, self-applied PBM therapy at the clinic or at home was found effective for resolving OM with rapid relief of related pain, as well as for accelerating healing in post-operative non-healing wounds and dermatitis, with no reported adverse events.

The treatment protocol was followed by the patients after a short explanation without difficulty. The extraoral/over skin treatment that was applied by the patients themselves was not painful and did not require time from the staff. The PBM home-use device itself is hand-held, lightweight, and the instructions are self-explanatory. However, the intra-oral application is not convenient due to the device configuration.

PBM is already part of the official guidelines for the treatment of $\mathrm{OM}$ and is routinely used at hematological 
Fig. 1 Oral mucositis. Case \#2. Severe oral mucositis (World Health Organization grade 3) on palate and lips (a and $\mathbf{b}$, respectively) that led to halting chemotherapy. White and black arrows indicating palatial and lip lesions. After 5 treatments (c), complete healing of the palate and lips. Patient was then able to continue chemotherapy. For clinical details, see Table 1

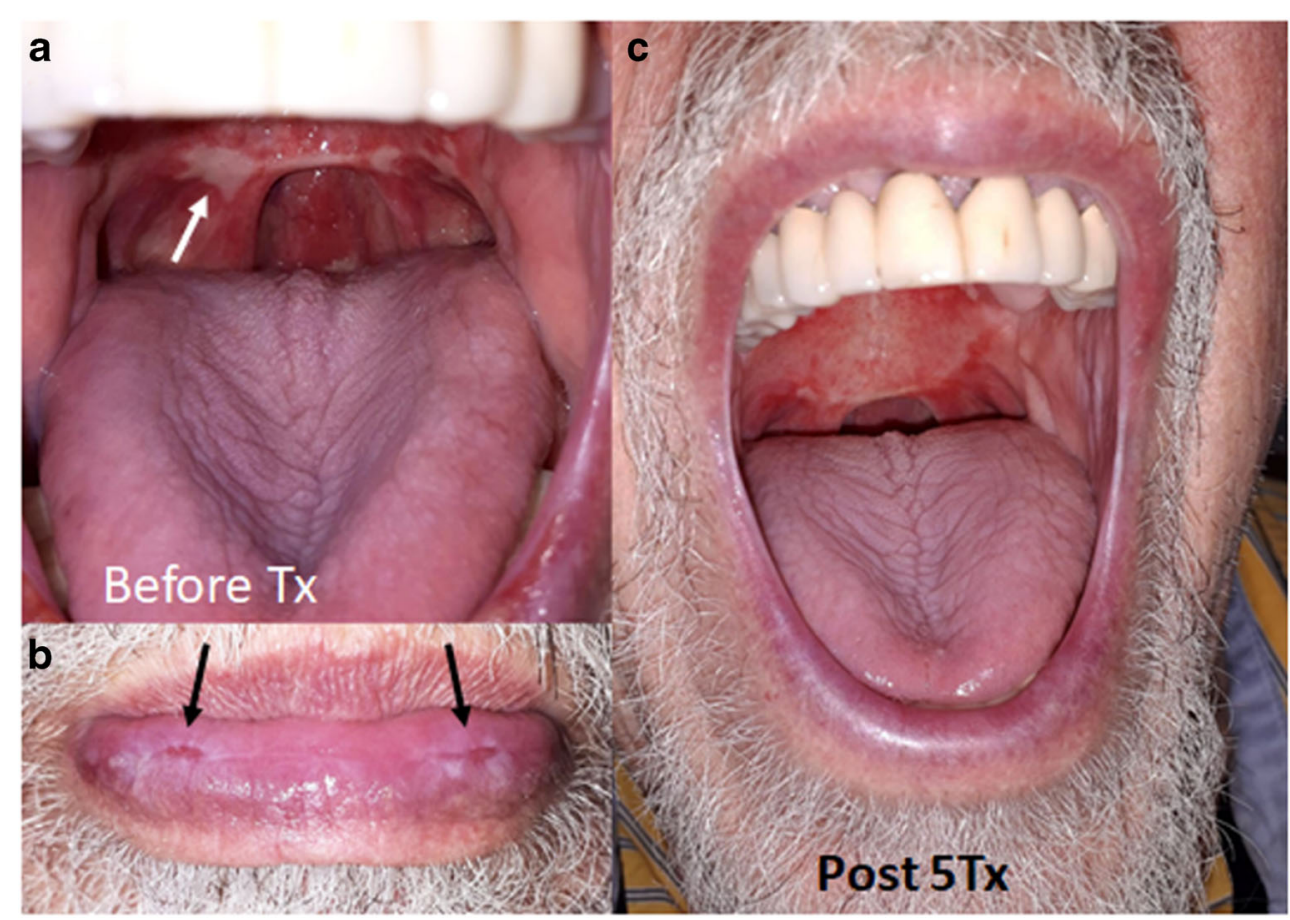

and oncological clinics [11, 12, 18]. Clinical studies also support the use of PBM for other toxicities induced by chemotherapy/radiotherapy including lymphedema, radiodermatitis, osteonecrosis of the jaw, and peripheral neuropathy $[7,19,20]$ as well as oral manifestations of chronic graft versus host disease following allogeneic hematopoietic stem cell transplantation [21, 22]. However, all previous reports related to treatments performed by the professional medical team at the clinic. This is the first report to show beneficial effects related to supportive care in cancer patients with a self-applied PBM device. In addition to pain relief and accelerated healing in cases of established OM (case \#1 and case \#2), this device may also allow a pre-conditioning period as well as convenient at-home prophylaxis treatments as done by patient \#2. A personal home treatment enables frequent treatments with a minimal number of additional visits to the hospital which is of high importance with regard to patient's convenience, compliance, and safety, for example, due to the need to restrict the

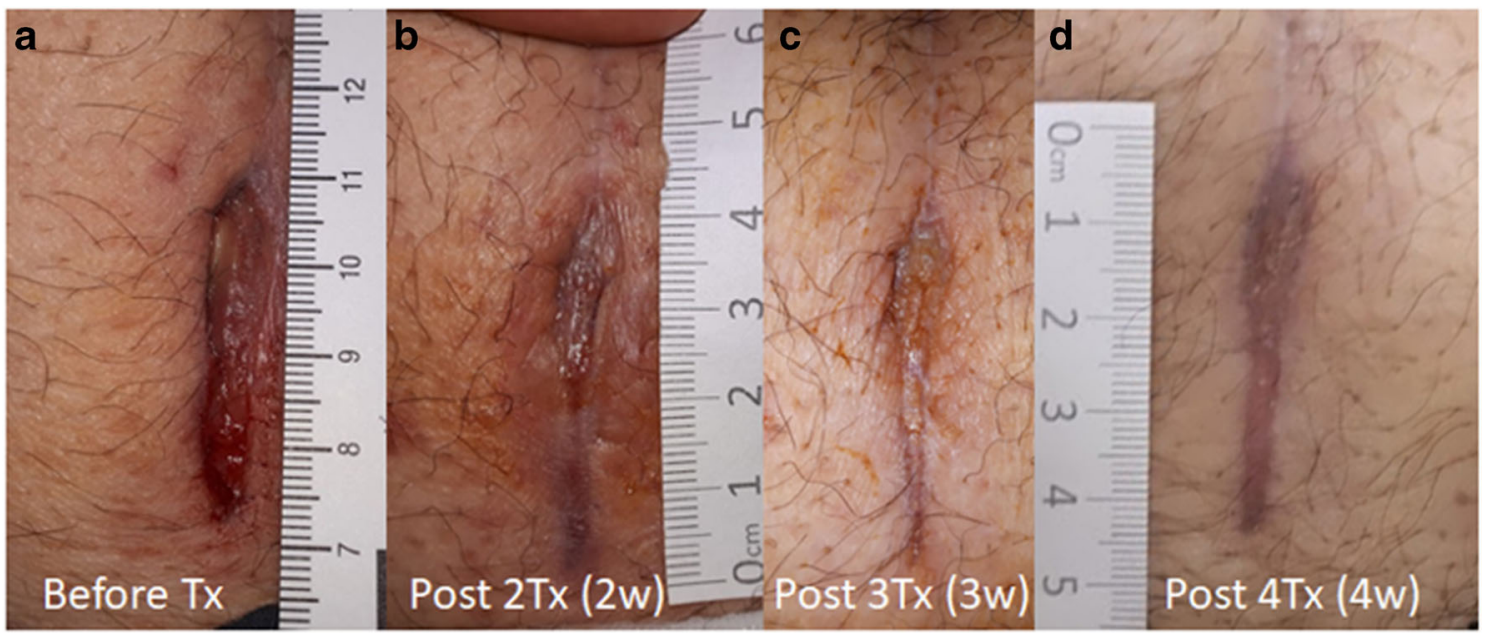

Fig. 2 Wound dehiscence. Case \#3. Surgical site infection following Hartmann procedure resulted in wound déhiscence (a). Two weeks after the first treatment, full granulation (b), and 1 week later the wound was completely epithelialized (c-d). For clinical details, see Table 1 
Fig. 3 Dermatitis. Case \#6. Skin irritation in groin area (a). The wound considerably improved after 4 daily photobiomodulation treatments (b) and completely healed within 2 weeks (c). For clinical details, see Table 1

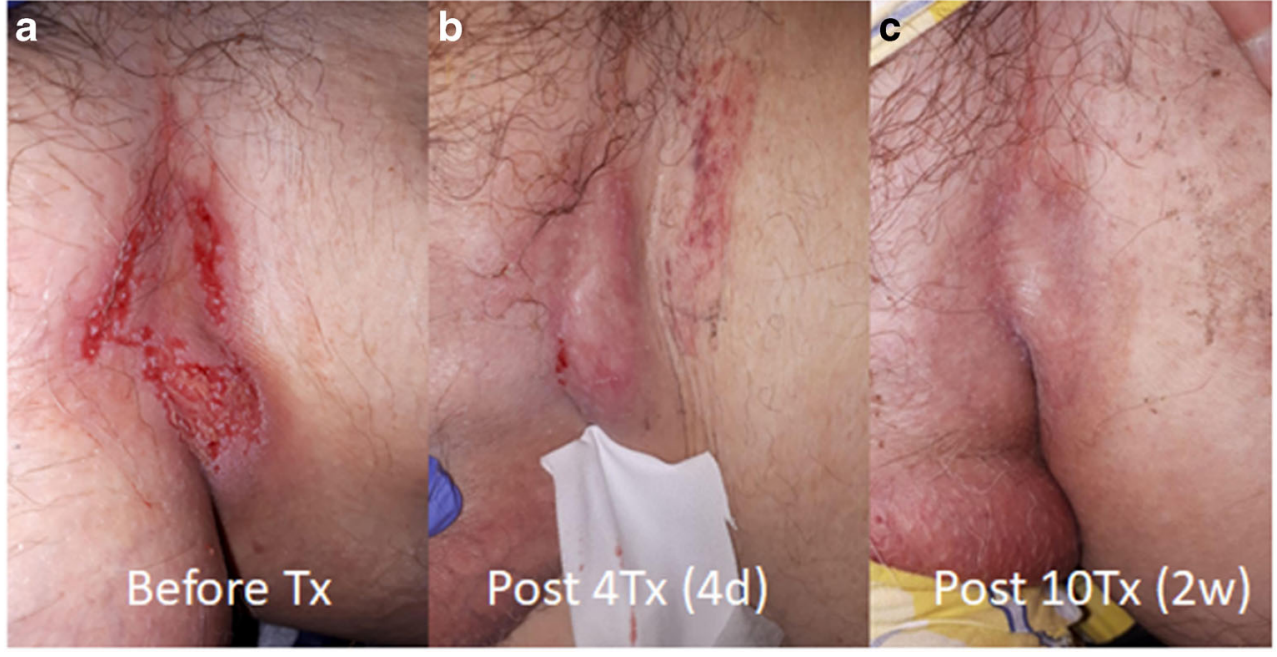

Table 1 Summary of cases

Medical background $\quad$ Treatment Main effect

Oral mucositis - protocol*: - daily: extraoral $3 \mathrm{~min}$; intraoral $2 \mathrm{~min}$; twice a day: lymph nodes $1 \mathrm{~min}$

Case\#1:

55 y.o., female, diffuse large B cell lymphoma, mucositis on the nose and the mouth induced by R-CHOP and MTX CT and 36 Gy radiation therapy to the mandible (because of jaw involvement). Unable to eat/drink (WHO grade 4). Treated with lidocaine mouth rinse, and acetaminophen $500 \mathrm{mg} / 4 \mathrm{~h}$ for pain. During the 3rd week of the RT, the patient initialized daily 30-minute PBM treatments.

Case\#2:

71 y.o., male, metastatic renal cell carcinoma, had to stop CT because of severe OM (WHO grade 3; Fig. 1).
$7 \mathrm{Tx}, 30$ min per session

Extraoral: each cheek, nose; Intraoral: under lip, anterior, and mid tongue; soft palate, additional 1 min treatment directly on lesions including tongue and left cheek; Lymph: $3 \mathrm{X}$ cervical lymph nodes (3 applications required to cover area) on each side

$5 \mathrm{Tx}, 20$ min per session

Extraoral: each cheek; Intraoral: anterior and mid tongue; soft palate, inner surface of lips; Lymph: $2 \mathrm{X}$ cervical lymph nodes on each side, $1 \mathrm{X}$ thoracic duct/right lymphatic duct

Preventive protocol, $10 \mathrm{~min}$ per session: $1 \mathrm{~min}$ intraoral, and 2 min extraoral.
Post-surgical wounds - protocol*: wound bed $0.5 \mathrm{~min}$; wound margins $2.5 \mathrm{~min}$; tunnel $4 \mathrm{~min}$

\section{Case\#3:}

76 y.o., male, metastatic (obstructive) colon cancer, on CT and RT. Following Hartmann procedure, surgical site infection led to abdominal dehiscence. The wound was initially treated with NPWT including Pico and collagen/ORC and foam twice a week, but the wound failed to close during 4 weeks (Fig. 2). Case\#4:

67 y.o., female, perforated cecal cancer, a $4.3 \mathrm{~cm}^{2}$ abdominal wound after hemicolectomy. The abdominal wound was treated with collagen/ORC and silicon foam dressing, and NPWT, but failed to epithelialize. Unbearable pain $(\mathrm{NRS}=10)$ unsuccessfully treated with acetaminophen $500 \mathrm{mg} / 6 \mathrm{~h}$.

Case\#5:

\section{$5 \mathrm{Tx}, 6 \mathrm{~min}$ per session}

Wound bed: X1

Wound margins: X2

$6 \mathrm{Tx}, 16$ min per session

Wound bed: X2

Wound margins and inflamed staple: X6
After $2 \mathrm{Tx}$, patient was able to eat soft food (WHO grade 3 ) and pain was considerably reduced. After 3 Tx patient did not require analgesics or anesthetic mouth rinse. The detailed timeline is presented in Table 2.
OM considerably resolved within 3 days (WHO grade 1), able to resume CT a day later. Patient switched to preventive protocol. OM returned only once (WHO grade 2) (patient continued CT).
Baseline wound size $1.75 \mathrm{~cm}^{2}$

Two weeks after the first treatment, full granulation, and 1 week later the wound was completely epithelialized
Baseline wound size $4.3 \mathrm{~cm}^{2}$

After 3 Tx (1 week) no pain.

Complete epithelialization in 3 weeks. 
Table 1 (continued)

\begin{tabular}{|c|c|c|}
\hline Medical background & Treatment & Main effect \\
\hline $\begin{array}{l}57 \text { y.o., female, breast cancer and diabetes } \\
\text { mellitus type } 2 \text {. The patient treated with partial } \\
\text { mastectomy with axillary dissection and } \\
\text { immediate reconstruction and CT, hormonal } \\
\text { therapy and steroid. Seroma with a } 4.5 \mathrm{~cm} \\
\text { long tunnel and wound infection was } \\
\text { diagnosed 1-month post-surgery. Standard } \\
\text { wound care included hydroconductive wound } \\
\text { dressing failed to improve the wound. Breast } \\
\text { was tender and hard on palpation. }\end{array}$ & $\begin{array}{l}\text { Tunnel: X1 } \\
\text { Lymph: Axillary lymph nodes, thoracic duct } \\
\text { Additional treatment at home, Lymph twice a } \\
\text { day, wound/tunnel every other day, added } \\
\text { daily treatment over breasts }\end{array}$ & $\begin{array}{l}\text { Wound size reduced. Tunnel closed in } 6 \text { weeks. } \\
\text { After beginning the additional treatment at } \\
\text { home, resolved tenderness of breast. }\end{array}$ \\
\hline \multicolumn{3}{|c|}{ Dermatitis - protocol*: wound bed $0.5 \mathrm{~min}$; wound margins $2 \mathrm{~min}$; twice a day: lymph nodes $1 \mathrm{~min}$} \\
\hline $\begin{array}{l}\text { Case\#6**: } \\
71 \text { y.o., male, metastatic renal cell carcinoma, } \\
\text { developed CT-related dermatitis with desqua- } \\
\text { mation in groin area (Fig. } 3 \text { ). The irritated skin } \\
\text { was delicate and easily bled upon simple } \\
\text { cleaning. }\end{array}$ & $\begin{array}{l}10 \mathrm{Tx}, 12 \text { min per session } \\
\text { Wound bed: X4 } \\
\text { Wound margins: } \mathrm{X} 4 \\
\text { Lymph: inguinal }\end{array}$ & $\begin{array}{l}\text { Groin wound improved after } 4 \text { days, completely } \\
\text { healed in } 2 \text { weeks. The patient continued CT } \\
\text { all the while. }\end{array}$ \\
\hline
\end{tabular}

$C T$ chemotherapy, $M T X$ methotrexate, min minute, $N R S$ numerical rating scale, with range of 0 to 10 , for self-assessment of pain, $N P W T$ negativepressure wound therapy, $O M$ oral mucositis, $O R C$ oxidized regenerated cellulose, $P B M$ photobiomodulation, $R$-CHOP rituximab, cyclophosphamide, doxorubicin, vincristine, and prednisone, $R T$ radiation therapy, $T x$ treatment, WHO World Health Organization, y.o. year old. *All photobiomodulation treatments were conducted using B-Cure Laser Pro (Good Energies Ltd., Haifa, Israel) with the following parameters: wavelength, $808 \mathrm{~nm}$; power, 250 $\mathrm{mW}\left(55.6 \mathrm{~mW} / \mathrm{cm}^{2}\right)$; frequency, $15 \mathrm{KHz}, 1.1 \mathrm{~J} / \mathrm{cm}^{2} / \mathrm{min}$; spot size, $4.5 \times 1.0 \mathrm{~cm}^{2}$. **Cases \#2 and \#6 are the same patient

patient exposure to the hospital environment in light of coronavirus disease (COVID-19) or other potential infection in immunocompromised cancer patients.

PBM was shown to be beneficial for acceleration of healing of both acute and chronic wounds [5] based on its ability to enhance the microcirculatory flow [23] and to stimulate the mitochondria energetic state even in stressed conditions [24, 25]. Particularly, the PBM protocol (and the same device) used in this study was previously reported to accelerate healing of diabetic foot ulcers $[15,16]$ and to prevent post-laminectomy scar formation when used as a preventive measure [26]. Other protocols were shown to be beneficial for prevention and accelerate healing in cases of wound dehiscence [27, 28]. However, dehiscence related to chemotherapy is particularly challenging, and therefore, the beneficial effect presented here warrants special attention.

In summary, side-effects induced by cancer therapy have a detrimental effect on the patient well-being and may delay or even prevent the patients from completing treatment regimens. PBM is already an established tool for prevention of OM. The advent of a self-applied personal PBM treatment with easy to apply protocols for a variety of side-effects makes this technology an important accessible and safe supportive care option.

Table 2 Case \#1 detailed timeline of treatments and outcomes

\begin{tabular}{llll}
\hline Date & \# Tx & Pain* and medication & Condition \\
\hline D0 & Baseline & 10/10, acetaminophen, rinse & Unable to eat/drink (WHO grade 4) \\
D1** & Post Tx1 & 8/10, acetaminophen, rinse & \\
D2 & Post Tx2 & 6/10, no acetaminophen, rinse & Able to eat (soft) food (WHO grade 2) \\
D3 & Post Tx3 & 3/10, no acetaminophen, no rinse & \\
D4 & Post Tx4 & No pain & \\
D7 & Post Tx7 & No pain & Able to eat (WHO grade 2), small nasal ulcers, still no taste (dysgeusia)
\end{tabular}

$D$ day, $T x$ treatment, $W H O$ World Health Organization

* Level of pain by numerical rating scale $[0=$ no pain, to $10=$ most intense pain imaginable $] /$ maximum pain

**Last chemotherapy/radiotherapy session 
Author contribution LG and RR have both substantially contributed to the conception, acquisition, and interpretation of the data of the work. YZ contributed to interpretation and writing of the work. All authors drafted the work together, revised it critically for important intellectual contents, and gave their final approval for the version to be published.

Funding The devices were given on loan by the manufacturer, Good Energies Ltd. The company was not involved in treatment, evaluation, documentation, or writing this manuscript.

\section{Declarations}

Ethics approval and consent to participate The Scarborough Health Network Research Ethics Board has reviewed the work and considers it a quality improvement project that does not require REB approval TCPS2 requirements (July 8, 2019, attached as a supplementary document). All patients consented to the use of their information for scientific purposes according to the institute guidelines. This consent was documented in their personal files. Both patients that appear in the figures are deceased.

Consent for publication The information in the figures including legends was anonymized and it is not possible to identify the person in the image.

Competing interests $\mathrm{RR}$ and $\mathrm{YZ}$ declare no competing interests. LG was paid as a consultant to give recommendations for treatment protocols.

\section{References}

1. Sonis ST (2009) Mucositis: the impact, biology and therapeutic opportunities of oral mucositis. Oral Oncol 45:1015-1020

2. Deptula M, Zielinski J, Wardowska A, Pikula M (2019) Wound healing complications in oncological patients: perspectives for cellular therapy. Postepy Dermatol Alergol 36:139-146

3. Chung H, Dai T, Sharma SK, Huang YY, Carroll JD, Hamblin MR (2012) The nuts and bolts of low-level laser (light) therapy. Ann Biomed Eng 40:516-533

4. Anders JJ, Lanzafame RJ, Arany PR (2015) Low-level light/laser therapy versus photobiomodulation therapy Photomed. Laser Surg 33:183-184

5. Gavish L (2013) Chapter 50: low-level laser therapy for wound healing. In: Hamblin M, Huang Y (eds) Handbook of Photomedicine. CRC Press, Boca Raton

6. Robijns J, Censabella S, Bulens P, Maes A, Mebis J (2017) The use of low-level light therapy in supportive care for patients with breast cancer: review of the literature. Lasers Med Sci 32:229-242

7. Zadik Y, Arany PR, Fregnani ER, Bossi P, Antunes HS, Bensadoun RJ, Gueiros LA, Majorana A, Nair RG, Ranna V, Tissing WJE, Vaddi A, Lubart R, Migliorati CA, Lalla RV, Cheng KKF, Elad S, Mucositis study group of the multinational association of supportive care in cancer/international society of oral O (2019) Systematic review of photobiomodulation for the management of oral mucositis in cancer patients and clinical practice guidelines. Support Care Cancer 27:3969-3983

8. Zecha JA, Raber-Durlacher JE, Nair RG, Epstein JB, Elad S, Hamblin MR, Barasch A, Migliorati CA, Milstein DM, Genot MT, Lansaat L, van der Brink R, Arnabat-Dominguez J, van der Molen L, Jacobi I, van Diessen J, de Lange J, Smeele LE, Schubert MM, Bensadoun RJ (2016) Low-level laser therapy/ photobiomodulation in the management of side effects of chemoradiation therapy in head and neck cancer: part 2: proposed applications and treatment protocols. Support Care Cancer 24: 2793-2805

9. de Pauli PM, Araujo ALD, Arboleda LPA, Palmier NR, Fonseca JM, Gomes-Silva W, Madrid-Troconis CC, Silveira FM, Martins MD, Faria KM, Ribeiro ACP, Brandao TB, Lopes MA, Leme AFP, Migliorati CA, Santos-Silva AR (2019) Tumor safety and side effects of photobiomodulation therapy used for prevention and management of cancer treatment toxicities. A systematic review. Oral Oncol 93:21-28

10. Zadik Y (2019) Photobiomodulation for the palliation of oral mucositis in cancer patients: the future is here. Future Oncol 15:36473649

11. NICE: National Institute for Health and Care Excellence U (2018) Interventional procedures guidance [IPG615]: low-level laser therapy for preventing or treating oral mucositis caused by radiotherapy or chemotherapy. In: Editor (ed)^(eds) Book Interventional procedures guidance [IPG615]: Low-level laser therapy for preventing or treating oral mucositis caused by radiotherapy or chemotherapy, City

12. Elad S, Cheng KKF, Lalla RV, Yarom N, Hong C, Logan RM, Bowen J, Gibson R, Saunders DP, Zadik Y, Ariyawardana A, Correa ME, Ranna V, Bossi P, Mucositis guidelines leadership group of the multinational association of supportive care in $\mathrm{C}$, International Society of Oral O (2020) MASCC/ISOO clinical practice guidelines for the management of mucositis secondary to cancer therapy. Cancer 126:4423-4431

13. Gavish L, Houreld NN (2019) Therapeutic efficacy of home-use photobiomodulation devices: a systematic literature review. Photobiomodul Photomed Laser Surg 37:4-16

14. Gileva OS, Libik TV, Chuprakov MA, Yakov AY, Mirsaev FZ (2017) Conference proceedings: B-cure laser dental pro technology for prevention and treatment of peri-implant mucositis In: Editor (ed $)^{\wedge}$ (eds) Book conference proceedings: B-cure laser dental pro technology for prevention and treatment of peri-implant mucositis AIP Publishing, City, pp. 020021-020021-020026

15. Merigo E, Lixin T, Zhao Z, Rocca J, Fornaini C (2020) Autoadministered photobiomodulation on diabetic foot ulcers treatment: a new way to manage it? Case Rep Med. https://doi.org/10.1155/ 2020/7428472

16. Raizman R, Gavish L (2020) At-home self-applied photobiomodulation device for the treatment of diabetic foot ulcers in adults with type 2 diabetes: report of 4 cases. Can J Diabetes 44 : 375-378

17. Gagnier JJ, Kienle G, Altman DG, Moher D, Sox H, Riley D, Group C (2013) The CARE guidelines: consensus-based clinical case reporting guideline development. BMJ Case Rep 2013. https:// doi.org/10.1136/bcr-2013-201554

18. Treister NS, London WB, Guo D, Malsch M, Verrill K, Brewer J, Margossian S, Duncan C (2016) A feasibility study evaluating extraoral photobiomodulation therapy for prevention of mucositis in pediatric hematopoietic cell transplantation. Photomed Laser Surg 34:178-184

19. Bensadoun RJ (2018) Photobiomodulation or low-level laser therapy in the management of cancer therapy-induced mucositis, dermatitis and lymphedema. Curr Opin Oncol 30:226-232

20. Bensadoun RJ, Epstein JB, Nair RG, Barasch A, Raber-Durlacher JE, Migliorati C, Genot-Klastersky MT, Treister N, Arany P, Lodewijckx J, Robijns J, World Association for Laser T (2020) Safety and efficacy of photobiomodulation therapy in oncology: a systematic review. Cancer Med 9:8279-8300

21. Epstein JB, Raber-Durlacher JE, Epstein GL, Hazenberg MD, Tzachanis D, Spielberger RT (2020) Chronic oral graft-versushost disease: induction and maintenance therapy with photobiomodulation therapy. Support Care Cancer. https://doi. org/10.1007/s00520-020-05626-3 
22. Epstein JB, Raber-Durlacher JE, Lill M, Linhares YP, Chang J, Barasch A, Slief RI, Geuke M, Zecha JA, Milstein DM, Tzachanis D (2017) Photobiomodulation therapy in the management of chronic oral graft-versus-host disease. Support Care Cancer 25:357-364

23. Gavish L, Hoffer O, Rabin N, Halak M, Shkilevich S, Shayovitz Y, Weizman G, Haim O, Gavish B, Gertz SD, Ovadia-Blechman Z (2020) Microcirculatory response to photobiomodulation - why some respond and others do not: a randomised controlled study. Lasers Surg Med 52:863-872

24. Gavish L, Asher Y, Becker Y, Kleinman Y (2004) Low level laser irradiation stimulates mitochondrial membrane potential and disperses subnuclear promyelocytic leukemia protein. Lasers Surg Med 35:369-376

25. Gavish L, Gilon D, Beeri R, Zuckerman A, Nachman D, Gertz SD (2020) Photobiomodulation and estrogen stabilize mitochondrial membrane potential in angiotensin II challenged porcine aortic smooth muscle cells. J Biophotonics 14(1):e202000329

26. Holanda V, Pereira B, Ferreira K, Greiffo F, Oliveira J, Franca C, Silva D, Ontaneda D, Pinto N, Chavantes MC (2014) Abstract:
Low Level Laser Therapy prevents complications post laminectomy. In: ASLMS: the 34th Annual Conference of the American Society for Lasers Medicine and Surgery, Phoenix, Arizona

27. Dixit S, Maiya A, Umakanth S, Borkar S (2013) Photobiomodulation of surgical wound dehiscence in a diabetic individual by low-level laser therapy following median sternotomy. Indian J Palliat Care 19:71-75

28. Lima AC, Fernandes GA, de Barros AR, Gonzaga IC, de Oliveira RA, Nicolau RA (2017) Photobiomodulation (laser and LED) on sternotomy healing in hyperglycemic and normoglycemic patients who underwent coronary bypass surgery with internal mammary artery grafts: a randomized, double-blind study with follow-up. Photomed Laser Surg 35:24-31

Publisher's note Springer Nature remains neutral with regard to jurisdictional claims in published maps and institutional affiliations. 